\title{
Application of a complete blood count to screening lethargic and anorectic cats for pancreatitis
}

\author{
Magdalena Maria Krasztel ${ }^{1}$, Michał Czopowicz ${ }^{1 *}$, Olga Szaluś-Jordanow², Agata Moroz ${ }^{1}$, Marcin Mickiewicz and
} Jarosław Kaba'

\begin{abstract}
Background: Feline pancreatitis (FP) is an important health problem of cats. Its diagnostics is based on the combination of quantification of serum pancreatic lipase immunoreactivity (fPLI) and abdominal ultrasonography (AUS). These modalities allow for establishing highly specific diagnosis, however they are relatively expensive and time-consuming. On the other hand, a screening test of high sensitivity which would allow to rule out FP on the first visit without a considerable increase of costs would be clinically useful. To evaluate accuracy of nonspecific inflammatory biomarkers based on complete blood count (CBC) in diagnosing FP 73 client-owned cats with signs of lethargy and reduced appetite lasting for at least 2 days before presentation were enrolled in the cross-sectional study. They were examined with $\mathrm{fPLI}$ assay and AUS and classified as cats with very low risk of FP when $\mathrm{fPLI} \leq 3.5 \mu \mathrm{g} / \mathrm{L}$ and AUS negative for FP, or as cats with increased risk of FP in the case of any other combination of results. Then, 7 various CBC measurements were measured in each cat and linked to the risk of FP using the multivariable logistic regression.
\end{abstract}

Results: Five $\mathrm{CBC}$ measurements turned out to be significantly associated with the risk of FP - total leukocyte count $\left(W B C ;\right.$ crude odds ratio $\left.\left(\mathrm{OR}_{\text {crude }}\right)=12.2 ; \mathrm{Cl} 95 \%: 1.52,98.5\right)$, total neutrophil count $\left(\mathrm{OR}_{\text {crude }}=5.84 ; \mathrm{Cl} 95 \%: 1.22,27.9\right)$, band neutrophil count $\left(B N C ; \mathrm{OR}_{\text {crude }}=6.67 ; \mathrm{Cl} 95 \%\right.$ : 1.98, 22.4), neutrophil-to-lymphocyte ratio $\left(\mathrm{OR}_{\text {crude }}=3.68 ; \mathrm{Cl} 95 \%\right.$ : $1.25,10.9)$, and eosinophil count ( $\left.E C ; O R_{\text {crude }}=0.34 ; C I 95 \%: 0.12,0.96\right)$. The model based on WBC, BNC, and EC proved to have at least fair diagnostic potential (area under ROC curve 82.7\%; Cl 95\%: 72.8\%, 92.5\%). When WBC < 18G/L, $\mathrm{BNC}<0.27 \mathrm{G} / \mathrm{L}$, and $\mathrm{EC}>0.3 \mathrm{G} / \mathrm{L}$ was considered as a negative result, and any other combination as the positive result, the CBC model had high sensitivity (91.8\%; Cl 95\%: 80.8\%, 96.8\%) at a relatively low specificity (58.3\%; Cl 95\%: $38.8 \%, 75.5 \%)$.

Conclusion: The combination of three CBC measurements is an immediately available and fairly accurate screening method for identification of lethargic and anorectic cats with increased risk of FP.

Keywords: Abdominal ultrasonography, Band neutrophil count, Eosinophil count, Feline pancreatic lipase immunoreactivity, fPLI, Neutrophil-to-lymphocyte ratio, White blood cell count

\footnotetext{
*Correspondence: mczopowicz@gmail.com

${ }^{1}$ Division of Veterinary Epidemiology and Economics, Institute

of Veterinary Medicine, Warsaw University of Life Sciences-SGGW,

Nowoursynowska 159c, 02-776 Warsaw, Poland

Full list of author information is available at the end of the article
}

\section{Background}

Feline pancreatitis (FP) is a relatively common disease whose etiology usually remains unknown, clinical manifestation is nonspecific, diagnostics difficult and expensive, and prognosis guarded [1-5]. Lethargy and reduced appetite or anorexia are most common clinical signs, followed by vomiting, weight loss, and less often diarrhea 
[5]. Even though, histologically three different FP forms are recognized - acute necrotizing pancreatitis, acute suppurative pancreatitis, and chronic pancreatitis [6,7], their similar clinical manifestation renders them barely distinguishable for a practitioner [8]. Therefore, the general term feline pancreatitis is commonly used [5].

At present the combination of clinical chemistry assays measuring pancreatic lipase immunoreactivity (serum pancreatic lipase immunoreactivity, fPLI) or activity (colorimetric 1,2-o-Dilauryl-Rac-Glycero-3-Glutaric Acid(6'-Methylresorufin) Ester (DGGR) assay) and abdominal ultrasonography (AUS) is a mainstay of clinical diagnostics of FP $[2,3,5,9]$. The estimates of diagnostic accuracy of these tests vary between studies. Diagnostic sensitivity (Se) and specificity (Sp) range from $50 \%$ to $80 \%$ and from $50 \%$ to $100 \%$, respectively, for fPLI [10-14] and from $25 \%$ to $80 \%$ and from $70 \%$ to $90 \%$, respectively, for AUS [10, $15,16]$. These discrepancies result from different cut-off values of the test (fPLI of 3.5 or $5.3 \mu \mathrm{g} / \mathrm{L} ; 1$ or 2 ultrasonographic changes indicative of FP), different severity of FP in a study population (mild or marked) as well as differences in the skills of AUS examiners and quality of ultrasound devices. Nevertheless, most of studies indicate that Sp outweighs Se [2, 3, 17]. Hence, unless a pretest probability of FP is very low, positive results of these tests tend to be more trustworthy than negative (i.e. positive predictive value, PPV outraces negative predictive value, NPV). This renders them rather confirmatory then screening tests. Therefore, an easily available and inexpensive screening method of high Se for an immediate identification of patients with increased risk of FP would be of value.

Various inflammatory biomarkers are used in human and veterinary medicine for tentative detection, or rather exclusion, of active inflammatory, infectious and neoplastic processes [18]. Of them biomarkers based on complete blood count (CBC) such as total (WBC) and differential leukocyte count, as well as neutrophilto-lymphocyte ratio (NLR) have been investigated in small animal veterinary medicine mainly due to their low cost and broad availability [19-23]. The presence or severity of pancreatitis in cats have been recently linked to NLR [22].

In this study, we evaluated the accuracy of a handful of $\mathrm{CBC}$ measurements in making the first-line decision whether a cat with lethargy and reduced appetite was at increased risk of FP.

\section{Results}

The study enrolled 73 adult castrated cats, 45 males (61.6\%) and 28 females (38.4\%), aged from 2 to 18 years with the median (IQR) of 10 (8 to 12 ) years. Age did not differ significantly between sexes $(p=0.750)$. Fifty eight cats $(79.5 \%)$ were domestic shorthair. Others were Siberian $(n=5,6.9 \%)$, British shorthair $(n=3,4.1 \%)$, Maine coon, Russian and Devon rex (each $n=2,2.7 \%$ ), and Siamese $(n=1,1.4 \%)$.

fPLI was normal $(\leq 3.5 \mu \mathrm{g} / \mathrm{L})$ in 33 cats $(45.2 \%)$ and elevated (>3.5 $\mu \mathrm{g} / \mathrm{L})$ in 40 cats $(54.8 \%)$ : from 3.6 to $5.3 \mu \mathrm{g} / \mathrm{L}$ in 8 cats and $>5.3 \mu \mathrm{g} / \mathrm{L}$ in 32 cats. At least one ultrasonographic feature of FP was detected in 26 cats (35.6\%). On the basis of those two diagnostic tests the risk of FP was determined as very low (fPLI $\leq 3.5 \mu \mathrm{g} / \mathrm{L}$ and normal appearance of the pancreas in AUS) in 24 cats (32.9\%) and increased (fPLI $>3.5 \mu \mathrm{g} / \mathrm{L}$ and/or abnormal appearance of the pancreas in AUS) in 49 cats (67.1\%). From among 49 cats with increased risk of FP only 17 cats (34.7\%) had both elevated fPLI and abnormal appearance of the pancreas in AUS. Twenty three cats (46.9\%) had elevated fPLI but normal appearance of the pancreas in AUS, and 9 cats (18.4\%) had abnormal appearance of the pancreas in AUS but normal fPLI.

Diabetes mellitus was present in 29 cats (39.7\%), suspected hepatopathy in 22 cats $(30.1 \%$; ALT [median (range)] - 307 (58-2322) U/L; TB - 18.8 (6.8-359.1) $\mu \mathrm{mol} / \mathrm{L})$, suspected acute kidney injury in 7 cats $(9.6 \%$; urea - $29.0(17.9-62.7) \mathrm{mmol} / \mathrm{L}$, creatinine - 353.6 $(256.4-1246) \mu \mathrm{mol} / \mathrm{L})$, and neoplastic disease in 3 cats (4.1\%) (malignant liver tumor, intestinal lymphoma, and disseminated pulmonary metastases from the mammary gland tumor). Hemoconcentration was observed in 23 cats (31.5\%; Ht - $0.38(0.16-0.51)$, TP - 73 (52-97) g/L, urea - $19.9(9.1-97.2) \mathrm{mmol} / \mathrm{L}$, creatinine - 168 (97.2256.4) $\mu \mathrm{mol} / \mathrm{L})$ and anemia in 10 cats $(13.7 \%$; $\mathrm{Ht}-0.24$ (0.16-0.26)). Vomiting was reported on admission in 37 cats $(50.7 \%)$ and diarrhea in 9 cats $(12.3 \%)$. Abdominal pain was detected by an attending veterinarian in 13 cats $(17.8 \%)$ and fever in 3 cats $(4.1 \%)$. Cats with increased risk of FP were significantly older $(p=0.038)$. All other demographic and clinical characteristics were evenly distributed between cats with very low and increased risk of FP (Table 1).

Numerical values of 7 CBC measurements and cutoff values used for their categorization are presented in Additional file 1.

The univariable analysis yielded $5 \mathrm{CBC}$ measurements significantly linked to an increased risk of $\mathrm{FP}$ - four positively associated: WBC, total neutrophil count (TNC), band neutrophil count (BNC), NLR, and one negatively associated: eosinophil count (EC) (Table 2).

Three $\mathrm{CBC}$ measurements proved to be significantly associated with increased risk of FP in the multivariable analysis (Table 3 ). The odds of being at increased risk of FP were 13-fold higher when WBC was $\geq 18 \mathrm{G} / \mathrm{L}$ and almost 5 -fold higherwhen $\mathrm{BNC}$ was $\geq 0.27 \mathrm{G} / \mathrm{L}$, while they were roughly 4 -fold lower when $\mathrm{EC}$ was $>0.3 \mathrm{G} / \mathrm{L}$. 
Table 1 Demographic and clinical characteristics of the study cats

\begin{tabular}{|c|c|c|c|}
\hline \multirow[t]{2}{*}{ Characteristic $^{\mathrm{a}}$} & \multicolumn{2}{|c|}{ Risk of feline pancreatitis } & \multirow[t]{2}{*}{$P$-value ${ }^{b}$} \\
\hline & Very low $(n=24)$ & Increased $(n=49)$ & \\
\hline \multicolumn{4}{|l|}{ Demographic characteristics } \\
\hline Age [years] - median, IQR, (range) & $10,7-11(2-15)$ & $11,9-13(2-18)$ & $0.038^{*}$ \\
\hline Sex-males & $14(58.3)$ & $31(63.3)$ & $0.685^{c}$ \\
\hline Breed - domestic shorthair & $3(12.5)$ & $12(24.5)$ & 0.218 \\
\hline \multicolumn{4}{|l|}{ Comorbidities } \\
\hline Diabetes mellitus & $9(37.5)$ & $20(40.8)$ & 0.785 \\
\hline Suspected hepatopathy & $5(20.8)$ & $17(34.7)$ & 0.216 \\
\hline Suspected acute kidney injury & $2(8.3)$ & $5(10.2)$ & $0.999^{d}$ \\
\hline Anemia & $2(8.3)$ & $8(16.3)$ & $0.481^{d}$ \\
\hline Hemoconcentration & $6(25.0)$ & $17(34.7)$ & 0.397 \\
\hline Hyperthyroidism & $1(4.2)$ & 0 & $0.319^{d}$ \\
\hline Neoplastic disease & 0 & $3(6.1)$ & $0.546^{\mathrm{d}}$ \\
\hline \multicolumn{4}{|l|}{ Clinical signs } \\
\hline Vomiting & $9(37.5)$ & $28(57.1)$ & 0.113 \\
\hline Diarrhea & $6(25.0)$ & $3(6.1)$ & $0.051^{d}$ \\
\hline Abdominal pain & $3(12.5)$ & $10(20.4)$ & 0.407 \\
\hline Fever & 0 & $3(6.1)$ & $0.546^{d}$ \\
\hline
\end{tabular}

* Significant at $a=0.05$

a Presented as $\mathrm{n}(\%)$ unless otherwise stated

${ }^{\mathrm{b}}$ Maximum likelihood G-test unless otherwise stated

cMann-Whitney $U$ test

${ }^{\mathrm{d}}$ Fisher exact test

Table 2 The univariable analysis of the association between categorized complete blood count (CBC) measurements and increased risk of feline pancreatitis (FP) in the study cats

\begin{tabular}{|c|c|c|c|c|}
\hline CBC-based inflammatory biomarker & Category & $\begin{array}{l}\text { Cats with increased risk of FP / all } \\
\text { cats in the category (\%) }\end{array}$ & $P$-value ${ }^{a}$ & $\begin{array}{l}\text { Crude odds ratio } \\
\left(\mathrm{OR}_{\text {crude }}\right)(\mathrm{Cl} 95 \%)\end{array}$ \\
\hline \multirow[t]{2}{*}{ Total leukocyte count $[\mathrm{G} / \mathrm{L}]$} & $\geq 18(n=18)$ & $17(94.4)$ & $0.002^{*}$ & $12.2(1.52,98.5)$ \\
\hline & $<18(n=55)$ & $32(58.2)$ & & \\
\hline \multirow[t]{2}{*}{ Total neutrophil count $[\mathrm{G} / \mathrm{L}]$} & $\geq 15(n=19)$ & $17(89.5)$ & $0.010^{*}$ & $5.84(1.22,27.9)$ \\
\hline & $<15(n=54)$ & $32(59.3)$ & & \\
\hline \multirow[t]{2}{*}{ Band neutrophil count [G/L] } & $\geq 0.27(n=32)$ & $28(87.5)$ & $0.001^{*}$ & $6.67(1.98,22.4)$ \\
\hline & $<0.27(n=41)$ & $21(51.2)$ & & \\
\hline \multirow[t]{2}{*}{ Lymphocyte count [G/L] } & $\geq 2.2(n=36)$ & $21(58.3)$ & 0.113 & $0.45(0.17,1.23)$ \\
\hline & $<2.2(n=37)$ & $28(75.6)$ & & \\
\hline \multirow[t]{2}{*}{ Eosinophil count $[\mathrm{G} / \mathrm{L}]$} & $>0.3(n=36)$ & $20(55.6)$ & $0.037^{*}$ & $0.34(0.12,0.96)$ \\
\hline & $\leq 0.3(n=37)$ & $29(78.4)$ & & \\
\hline \multirow[t]{2}{*}{ Monocyte count $[G / L]$} & $\geq 0.15(n=12)$ & $11(91.6)$ & 0.089 & $6.95(0.84,57.3)$ \\
\hline & $<0.15(n=61)$ & $38(62.3)$ & & \\
\hline \multirow[t]{2}{*}{ Neutrophil-to-lymphocyte ratio [1/1] } & $\geq 4.7(n=33)$ & $27(81.8)$ & $0.013^{*}$ & $3.68(1.25,10.9)$ \\
\hline & $<4.7(n=40)$ & $22(55.0)$ & & \\
\hline
\end{tabular}

*Significant at $a=0.05$

${ }^{\text {a }}$ The maximum likelihood G-test 
Table 3 Multiple logistic regression model based on a complete blood count (CBC) aiming to identify these anorectic and lethargic cats in which the risk of pancreatitis is increased

\begin{tabular}{lllll}
\hline CBC measurements & Regression coefficient (SE) & Wald's statistics & $\boldsymbol{P}_{\text {-value }}$ & OR $_{\text {adj }}$ (CI 95\%) \\
\hline Intercept & $0.52(0.46)$ & - & - & - \\
WBC $\geq 18 \mathrm{G} / \mathrm{L}$ & $2.59(1.12)$ & 5.32 & 0.021 & $13.3(1.47,121)$ \\
$\mathrm{BNC} \geq 0.27 \mathrm{G} / \mathrm{L}$ & $1.59(0.67)$ & 5.56 & 0.018 & $4.88(1.31,18.2)$ \\
$\mathrm{EC}>0.3 \mathrm{G} / \mathrm{L}$ & $-1.49(0.61)$ & 5.98 & 0.014 & $0.23(0.07,0.74)$ \\
\hline
\end{tabular}

BNC Band neutrophil count, Cl 95\% 95\% confidence interval, EC Eosinophil count [G/L], OR adj Adjusted odds ratio, SE Standard error, WBC Total leukocyte count [G/L]

The model based on 3 CBC measurements (henceforth referred to as CBC model) fit the data well (H\&L $\mathrm{X}^{2}=3.04, p=0.551$; Nagelkerke's pseudo- $R^{2}=0.40$ ).

The CBC model had fair to good discriminatory potential $\quad$ (AUROC $=82.7 \%$; CI 95\%: 72.8\%, 92.5\%; $p<0.001$ ), which was significantly higher than a discriminatory potential of each $\mathrm{CBC}$ measurement separately (Fig. 1): mean difference of AUROC between the $\mathrm{CBC}$ model and: $\mathrm{WBC}=24.8 \%$ (CI 95\%: $10.9 \%, 38.7 \%$; $p=0.001)$; $\mathrm{BNC}=11.0 \%$ (CI 95\%: 1.6\%, 20.4\%; $p=0.022$ ); $\mathrm{EC}=22.0 \%$ (CI 95\%: 7.4\%, 36.3\%; $p=0.003$ ).

At the optimal cut-off value $\geq 0.628$ (i.e. result of the $\mathrm{CBC}$ model considered as negative only when $\mathrm{WBC}<18 \mathrm{G} / \mathrm{L}, \mathrm{BNC}<0.27$ and $\mathrm{EC}>0.3 \mathrm{G} / \mathrm{L})$ the $\mathrm{CBC}$ model's Se was 91.8\% (CI 95\%: 80.8\%, 96.8\%), and Sp was 58.3\% (CI 95\%: 38.8\%, 75.5\%), while positive (LR+) and negative (LR-) likelihood ratios were 2.2 (CI 95\%: 1.4, 3.6) and 0.14 (CI 95\%: 0.05, 0.38), respectively. The accuracy measures of the $\mathrm{CBC}$ model at other cut-off values (i.e. at other combinations of the three $\mathrm{CBC}$ measurements included in the CBC model) are presented in Table 4.

\section{Discussion}

In this study we developed a 3-element logistic regression model (CBC model) which has at least fair accuracy (confirmed by the lower 95\% confidence limit above the threshold of $70 \%$ ) in distinguishing between symptomatic cats with very low or increased risk of FP. The CBC model

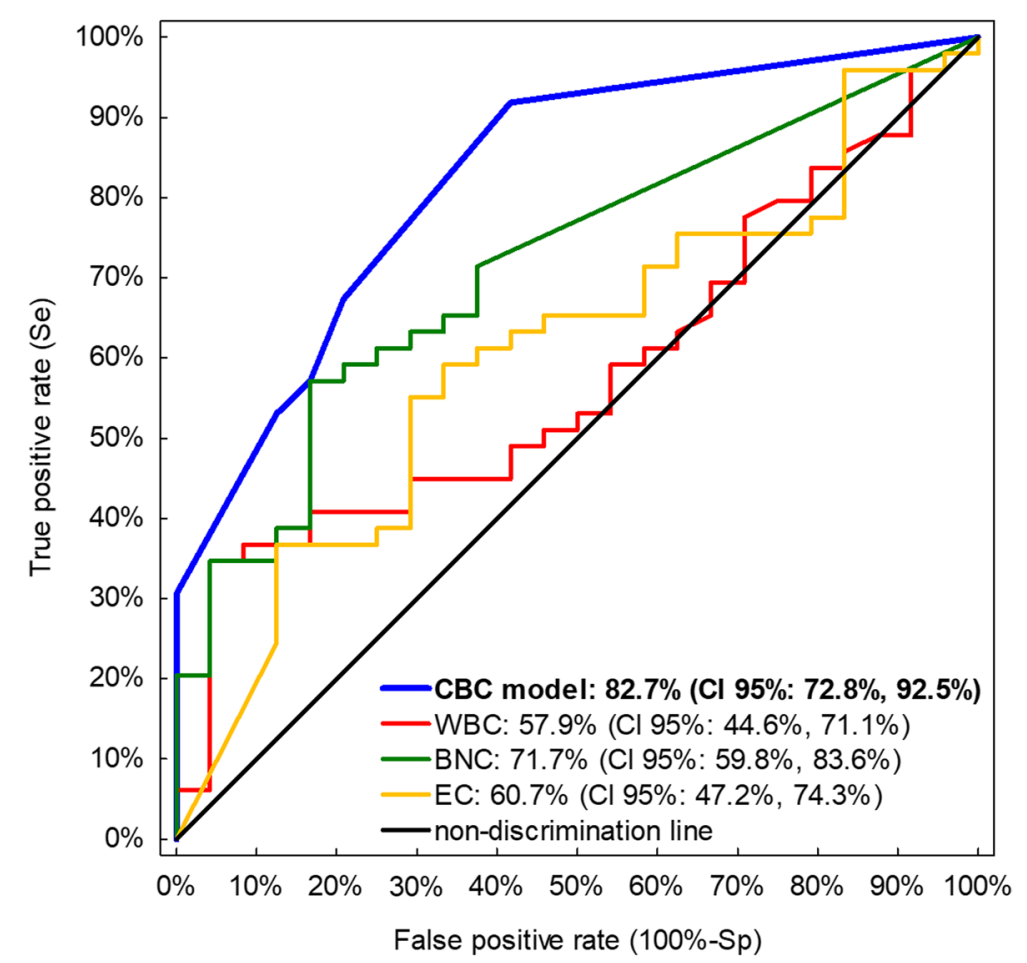

Fig. 1 ROC curves of the logistic model developed using three complete blood count (CBC) measurements as well as of each CBC measurement: WBC - total leukocyte count $[G / L], B N C$ - band neutrophil count $[G / L], E C$ - eosinophil count $[G / L]$ ). Area under ROC curve with $95 \%$ confidence interval (CI 95\%) presented for each CBC measurement 
Table 4 Accuracy of complete blood count (CBC) model at various cut-off values i.e. at various combinations of CBC measurements included in the model. The optimal cut-off value determined on the basis of the highest Youden's index $(J)$ highlighted (the combination above it i.e. $W B C<18 \mathrm{G} / \mathrm{L}, \mathrm{BNC}<0.27 \mathrm{G} / \mathrm{L}$, and $\mathrm{EC}>0.3 \mathrm{G} / \mathrm{L}$ is the only to be considered as negative)

\begin{tabular}{|c|c|c|c|c|c|c|c|c|}
\hline \multicolumn{3}{|c|}{$\begin{array}{l}\text { Combinations of } \mathrm{CBC} \\
\text { measurements }\end{array}$} & \multirow[t]{2}{*}{$\begin{array}{l}\text { Result of } \\
\text { CBC model }\end{array}$} & \multicolumn{5}{|c|}{ Accuracy measures } \\
\hline$W B C \geq 18$ & $\mathrm{BNC} \geq 0.27$ & $\mathrm{EC}>0.3$ & & Se (Cl 95\%) & $\mathrm{Sp}(\mathrm{Cl} 95 \%)$ & $\mathrm{LR}+(\mathrm{Cl}$ 95\%) & LR- (Cl 95\%) & $J$ \\
\hline No & No & Yes & 0.275 & - & - & - & - & - \\
\hline No & No & No & 0.628 & $91.8(80.8,96.8)$ & $58.3(38.8,75.5)$ & $2.2(1.4,3.6)$ & $0.14(0.05,0.38)$ & $50.2(39.4,61.0)$ \\
\hline No & Yes & Yes & 0.649 & $67.3(53.4,78.8)$ & $79.2(59.5,90.8)$ & $3.2(1.4,7.2)$ & $0.41(0.26,0.65)$ & $46.5(35.9,57.2)$ \\
\hline Yes & No & Yes & 0.835 & $57.1(43.3,70.0)$ & $83.3(64.1,93.3)$ & $3.4(1.4,8.7)$ & $0.51(0.36,0.74)$ & $40.5(30.1,50.9)$ \\
\hline No & Yes & No & 0.892 & $53.1(39.4,66.3)$ & $87.5(69.0,95.7)$ & $4.2(1.4,12.6)$ & $0.54(0.38,0.75)$ & $40.6(30.7,50.4)$ \\
\hline Yes & No & No & 0.957 & $30.6(19.5,44.5)$ & $100(86.2,100)$ & $+\infty$ & $0.69(0.58,0.84)$ & $30.6(24.0,37.2)$ \\
\hline Yes & Yes & Yes & 0.961 & $24.5(14.6,38.1)$ & $100(86.2,100)$ & $+\infty$ & $0.76(0.64,0.89)$ & $24.5(18.3,30.6)$ \\
\hline Yes & Yes & No & 0.991 & $6.1(2.1,16.5)$ & $100(86.2,100)$ & $+\infty$ & $0.94(0.87,1.01)$ & $6.1(2.7,9.5)$ \\
\hline
\end{tabular}

BNC Band neutrophil count [G/L], Cl 95\% 95\% confidence interval, EC Eosinophil count [G/L], JYouden's index [\%], LR+ Likelihood ratio of a positive result [1/1], LRLikelihood ratio of a negative result [1/1], Se Diagnostic sensitivity [\%], Sp Diagnostic specificity [\%], WBC Total leukocyte count [G/L]

has two important upsides. First, it is based on basic hematologic measurements (CBC-based inflammatory biomarkers: WBC, BNC, and $\mathrm{EC}$ ) which are routinely examined in virtually all apparently sick patients and are available from most veterinary laboratories within a few hours of blood collection. Secondly, CBC measurements were included in the $\mathrm{CBC}$ model as dichotomous variables categorized according to a cut-off value which was determined using sound statistical methodology (Youden's index-based criterion) [24, 25]. Three binary variables result in only $2^{3}=8$ potential combinations. As a result no calculations are necessary to apply the CBC model in daily practice. The only essential thing is the knowledge whether a given $\mathrm{CBC}$ measurement is below or above the cut-off value (Table 4).

CBC model is based on three types of leukocytes whose categorization corresponds to three well recognized changes observed in leukogram during the acute phase response: leukocytosis, left-shift, and eosinopenia [26-28]. Leukocytosis usually results from neutrophilia which develops easily and quickly in cats due to relatively high ratio (3:1) of marginal to circulating pool of neutrophils [26, 27, 29]. Immature band neutrophils are released from the bone marrow storage pool only when the inflammatory process leading to excessive migration of neutrophils to the affected tissues lasts for a longer time $[26,29]$. The fact that our study enrolled cats in which nonspecific clinical signs had been observed for at least 2 days explains important role of the left-shift in identifying cats with FP. However, it is possible that this feature may not be equally important if cats in which symptoms have begun more recently are being examined. In humans and many animal species neutrophilia is typically accompanied by lymphopenia during the acute inflammatory reaction [30]. In cats, however, it is not rare to observe the elevation of both neutrophils and lymphocytes $[27,28]$, especially when a concurrent excitement response triggered by stressful events such as physical examination, restraint and blood collection occur. In cats marked lymphocytosis is a prominent feature of the excitement response $[26,29,31]$. This may to some extent explain why lymphopenia was not included in the model and why NLR, which captures both neutrophilia and lymphopenia at the same time and therefore shows diagnostic usefulness in humans [32] and, to some extent, in dogs $[19,20]$, performed inferior to WBC in our study.

Our study aimed to identify CBC measurements which can be useful in making a tentative diagnosis of FP. Obviously, these measurements are not linked specifically to the pathological processes developing in the course of FP so they cannot be expected to perform as a highly accurate confirmatory test. In fact they may play role only in an immediate exclusion of FP, so they may work as a rapid screening test. However, to satisfy this expectation they must have sufficiently high Se so that a practitioner may thrust their negative result. Se of the $\mathrm{CBC}$ model at an optimal cut-off value was roughly $90 \%$, or at least $80 \%$ when we refer to the lower 95\% confidence limit. Even though, it may appear to be quite a high figure, we should focus on NPV, or more conveniently, on LR- which is independent of disease prevalence in a population [25]. LR- for CBC model was roughly 0.14 . It means that negative result is approximately 7 -fold more likely in a cat without than with FP. It is commonly accepted that LRshould be below 0.1 to be diagnostically useful i.e. negative result should be at least 10 -fold less likely to occur in a diseased than heathy individual [33]. On the other hand, plenty of tests of worse accuracy are commonly 
used in veterinary medicine, to mention only DGGRlipase assay which at a cut-off value of $26 \mathrm{U} / \mathrm{L}$ shows LRequal to 0.4 (calculated from the formula: LR- $=(1-\mathrm{Se}) /$ $\mathrm{Sp}=(1-0.667) / 0.786$; based on the figures reported in the study of Oppliger at al [13].). Detailed relationship between a pre-test probability of disease, LR, and a posttest probability of disease may be calculated by transformation of probabilities into odds [33,34] or from very convenient Fagan's nomogram $[35,36]$. Nevertheless, the rule of thumb says that the negative test result whose LRis 0.1 reduces pre-test probability by roughly $45 \%$ while LR- of 0.2 reduces it by roughly $30 \%$. CBC model is somewhere in between. However, in fact it is safer to stick to the upper 95\% confidence limit of LR- as tests usually perform worse in practice than they do in the population on which their accuracy has been assessed. Hence, when we assume that LR- is indeed closer to 0.4 and the pretest probability of $\mathrm{FP}$ in a cat presented to the veterinary clinic is $30 \%$ if this cat tests negative in CBC model its post-test probability will be roughly $15 \%$. Whether it is enough to justify practical use of $\mathrm{CBC}$ model is debatable. However, it is indisputable that applying $\mathrm{CBC}$ model costs nothing when $\mathrm{CBC}$ has already been done. On the other hand, we should be very cautious when using the $\mathrm{CBC}$ model as a confirmatory test. Even though, LR+ is very high (infinite) at certain cut-off values (Table 4) 49 cats used to determine Se (i.e. those classified as at increased risk of FP) were only moderately likely to have FP (as discussed in the next paragraph). Therefore, e.g. the cat with $\mathrm{WBC} \geq 18 \mathrm{G} / \mathrm{L}, \mathrm{BNC} \geq 0.27 \mathrm{G} / \mathrm{L}$, and $\mathrm{EC}$ $<0.3 \mathrm{G} / \mathrm{L}$ (the highest possible result of the CBC model) does not necessarily has FP. This cat simply appears to have an acute inflammatory reaction associated with at least one of the two tests (fPLI or AUS) positive for FP. Obviously, this result should make a practitioner examined this cat for FP using more specific methods, there are however many other diagnoses still probable in this cat.

The aforementioned limitation in the interpretation of positive results of the $\mathrm{CBC}$ model is associated with the fact that accuracy measures are of any value only if they have been estimated on the population of animals whose true heath status has been correctly determined [25, 37]. In our study we used two independent tests to diagnose FP - fPLI and AUS, at a cut-off value of $3.5 \mu \mathrm{g} / \mathrm{L}$ and at least one ultrasonographic feature of $\mathrm{FP}$, respectively. We had to make a decision of how to handle inconsistent results of the two tests as the agreement between these test has been shown to be low [38, 39]. The problem of inconclusive classification of animals in the studies investigating diagnostic accuracy of a test when no $100 \%$ accurate gold standard exists has been thoroughly reviewed [40]. Even though many approaches exist, all of them aim to eventually assign inconclusive individuals to one of the two groups (healthy or diseased) as evaluation of diagnostic accuracy is only possible when animals are classified according to a dichotomous manner. On the other hand, elimination of cats with inconsistent results would create two artificial groups of cats, extreme in terms of the likelihood of FP, with a huge gap between them. This would make these groups differ not only in terms of having FP but also in a general condition and many other features remaining beyond our control. As a result the accuracy measures of the $\mathrm{CBC}$ model would be falsely inflated. In our study we decided to apply fPLI and AUS in a parallel fashion which meant that any positive result indicated FP [25]. Diagnostic accuracy of each of these two tests at cut-off values applied in our study has so far been estimated several times before. In terms of fPLI Se and Sp were estimated at 61\% (CI 95\%: 36\%, $82 \%$ ) and 55\% (CI 95\%: 39\%, 70\%), respectively, on 60 cats [13], 65\% (CI 95\%: 43\%, 83\%) and 63\% (CI 95\%: 26\%, $90 \%)$, respectively, on 31 cats [12]. In an another study [14] Se was $74 \%$ for mild and $82 \%$ for marked FP, while Sp was $74 \%$. In terms of AUS Se was 84\% (CI 95\%: 60\%, 97\%) and Sp was 75\% (CI 95\%: 48\%, 93\%) on 35 cats [16]. Assuming Se and Sp of 65\% and 65\%, respectively, for fPLI and $85 \%$ and $75 \%$, respectively, for AUS, parallel testing procedure yields Se of $95 \%$ and Sp of $49 \%$. Then, assuming pre-test probability of $\mathrm{FP}$ of $50 \%$, the cat negative in both tests and on this basis considered as being at very low risk of FP in our study was $95 \%$ likely to be truly free from FP (therefore we referred to this group as cats at very low, not simply low, risk of FP). The cat positive in at least one of these tests was $65 \%$ likely to truly have FP, while a cat positive in both tests was $85 \%$ likely to truly have FP. Our CBC model is a screening test so we want to trust its negative result more than its positive result as the latter one will be further verified by tests more specific for FP. Therefore, in the study population we are more concerned with correct classification of cats as free from FP than as affected by FP. On the other hand, low $\mathrm{Sp}$ of a reference procedure tends to falsely reduce $\mathrm{Se}$ of an index test [41], so Se of the CBC model may be even higher than our estimations.

The most important limitation of our study is associated with the precision of $\mathrm{CBC}$ measurements used for the model development. While WBC was measured by modern automatic hematologic analyzers of high quality whose results are very likely to be highly repeatable, differential counts were determined manually by counting only 100 subsequent leukocytes. Such a method is prone to both human errors and random variability. For instance, CI 95\% for BN\% of 1\% estimated from 100 cells is from $0.2 \%$ to $5.4 \%$ [42], which markedly exceeds the cut-off value indicated by our analyses. Therefore, routine 
counting of 200 or even 300 leukocytes appears to be a reasonable recommendation (CI 95\% narrows down then to $0.1 \%$ to $2.8 \%$ and $0.1 \%$ to $1.9 \%$, respectively), although it would considerably extend the turnover time of blood samples. On the other hand, manual reviewing of blood films is the only method allowing to include band neutrophil count in the result. Our study substantiates the practical usefulness of estimating band neutrophil percentage and counts, which is especially important given the growing popularity of in-clinic instrumental hematologic analyzers. Another important downside of our analysis is the fact that we did not include the information on toxic changes in neutrophils. It was so since the evaluation of toxic neutrophils was not routinely provided by the veterinary laboratory in which our samples were examined. Toxic neutrophils are an important feature of $\mathrm{CBC}$ in acute inflammation caused by an enhanced turnover rate of neutrophils and incomplete neutrophil maturation in the bone marrow [26, 31]. Including this variable might have improved performance of the CBC model and it certainly warrants further investigation.

\section{Conclusions}

The combination of CBC measurements is an immediately available and fairly accurate screening method allowing to decide which cats presented with lethargy and anorexia have increased risk of FP.

\section{Methods}

This retrospective cross-sectional study comprised 73 cats presented to three veterinary clinics located in the Central Poland in years 2014-2020 and screened for FP using the commercial feline pancreatic lipase immunoreactivity (fPLI) assay and abdominal ultrasonography (AUS). Cats were enrolled in the study if their owners declared that a cat: 1) had lethargy and reduced appetite or anorexia for at least two previous days; 2) did not have any known chronic diseases; 3) had not been diagnosed with FP before; 4) had not been treated with glucocorticosteroids for a preceding month. All cats were examined by a specialist in small animal medicine. The blood sample was collected for a routine blood analysis and fPLI quantification. FPLI was measured $[\mu \mathrm{g} / \mathrm{L}]$ using ELISA based on monoclonal antibodies (Spec $\mathrm{fPL}^{\mathrm{TM}}$ ) in IDEXX Laboratories $\mathrm{GmbH}$ (Ludwigsburg, Germany). According to the manufacturer's recommendations fPLI was considered normal when $\leq 3.5 \mu \mathrm{g} / \mathrm{L}$ and elevated when $>3.5 \mu \mathrm{g} / \mathrm{L}$. AUS was performed by a veterinary radiologist with state-of-the art devices (MyLab 25 Gold, Esaote, Italy and HM70A, Samsung Electronics Ltd., UK). FP was ultrasonographically diagnosed if at least one of the following features was detected: pancreatomegaly (width of the pancreas $>10 \mathrm{~mm}$ ), irregular margins of the pancreas, hypoechoic parenchyma of the pancreas, hyperechoic surrounding mesenteric or fat, and peripancreatic free fluid. Hyperechoic pancreatic parenchyma indicative of fibrosis was also considered diagnostic for FP $[5,10$, 16]. Other diagnostic tests (thoracic radiography, echocardiography) were performed if necessary to make a definitive diagnosis.

Results of the two diagnostic tests used for detection of FP were interpreted according to a parallel manner [25] i.e. the risk of FP was considered as very low only when fPLI was normal $(\leq 3.5 \mu \mathrm{g} / \mathrm{L})$ and the appearance of the pancreas in AUS was normal. Otherwise, a cat was found to be at increased risk of FP.

The blood analysis was performed in a commercial veterinary laboratory and included routine complete blood count $(\mathrm{CBC})$, alanine aminotransferase (ALT) and alkaline phosphatase (ALP) activity, total protein (TP) and total bilirubin (TB) concentration as well as urea and creatinine concentration. Routine blood analysis was performed using Abacus Vet5 Hematology analyzer (Diatron MI Zrt., Hungary), Mythic 18 Vet (PZ Cormay S.A., Poland) and automatic photometric clinical chemistry analyzer BS-800 (Mindray Medical Poland). The differential leucocyte count was performed on thin and smooth peripheral blood smears stained with the May-Grünwald-Giemsa reagent (Adamed Pharma S.A., Poland). The smears were examined with a light microscope (Primo Star, Zeiss, Germany) under 100x magnification by a qualified technician and 100 nucleated cells were classified into six subpopulations: band neutrophils, segmented neutrophils, eosinophils, basophils, monocytes, and lymphocytes. Basophils were not included in further analyses as they were very rarely observed in manual blood smears and their correct identification was questionable.

Four clinical conditions were identified in cats using hematologic and biochemical criteria: anemia when $\mathrm{Ht}$ was <27\%; hemoconcentration when i) $\mathrm{Ht}$ was $>45 \%$ and TP was $>80 \mathrm{~g} / \mathrm{L}$ or ii) urea concentration was $>12 \mathrm{mmol} / \mathrm{L}$ at creatinine concentration $<250 \mu \mathrm{mol} / \mathrm{L}$; suspected acute kidney injury when creatinine concentration was $>250 \mu \mathrm{mol} / \mathrm{L}$ and urea concentration was $>12 \mathrm{mmol} / \mathrm{L}$, and suspected hepatopathy when ALT was $>200 \mathrm{U} / \mathrm{L}$ (without concurrent hyperthyroidism) or $\mathrm{TB}$ was $>17 \mu \mathrm{mol} / \mathrm{L}$ (without concurrent anemia) [43]. Diabetes mellitus was diagnosed based on polyuria and polydipsia in the medical history and fructosamine concentration $>400 \mu \mathrm{mol} / \mathrm{L}$, hyperthyroidism based on total thyroxine concentration $>65 \mathrm{nmol} / \mathrm{L}$, and neoplasms based on chest radiography, AUS, and ultrasoundguided fine needle biopsy. Fever was defined as rectal 
temperature $>39.5^{\circ} \mathrm{C}$. The presence of abdominal pain was subjectively assessed by a clinician.

Seven CBC measurements were analyzed in this study: WBC, total neutrophil count, band neutrophil count (BNC), eosinophil count (EC), monocyte count (MC), lymphocyte count (LC), and neutrophil-to-lymphocyte ratio (NLR).

No ethics commission approval for this study was required according to Polish legal regulations (the Act on the Protection of Animals Used for Scientific or Educational Purposes of 15 January 2015) as only routine diagnostic procedures essential given the clinical status of the cat were performed and the study was purely analytical. Nevertheless, an informed consent of each cat's owner for participation in the study was obtained.

Numerical variables were presented as the median, interquartile range (IQR) and range, and compared between two groups using the Mann-Whitney U test. Categorical variables were presented as a count (n) and percentage of the total number of animals in a given group. First, CBC measurements were transformed into dichotomous variables. The optimal cut-off value for dichotomization was determined using the highest Youden's index (J) criterion [24]. Then, the relationship between the categorized $\mathrm{CBC}$ measurements and the risk of FP was investigated using the maximum likelihood G-test or Fisher's exact test if the expected count in any cell of the two-by-two table was $<5$, and was expressed as the crude odds ratio $\left(\mathrm{OR}_{\text {crude }}\right)$.

$\mathrm{CBC}$ measurements which proved significantly linked $(\alpha=0.05)$ to the risk of FP in the univariable analysis were offered to the multiple logistic regression [44] according to the following formula:

$$
\mathrm{f}(\mathrm{P}=1)=\frac{1}{1+\mathrm{e}^{-\left(\mathrm{B}_{0}+\mathrm{B}_{\mathrm{n}} \times \mathrm{X}_{\mathrm{n}}\right)}}
$$

where e signified the Euler's number $(\approx 2.718), \mathrm{B}_{0}$ was the constant, $B_{n}$ were the regression coefficients of $C B C$ measurements $\left(\mathrm{X}_{\mathrm{n}}\right)$ entered in the initial model and evaluated using the backward stepwise elimination procedure. The magnitude of association between a CBC measurement and the risk of FP was expressed as the adjusted odds ratio $\left(\mathrm{OR}_{\mathrm{adj}}\right)$. The goodness-of-fit of the model was evaluated using the Hosmer-Lemeshow $\chi^{2}$ test $\left(H \& L X^{2}\right)$ and Negelkerke's pseudo- $R^{2}$ coefficient. Discriminatory potential of $\mathrm{CBC}$ measurements and logistic model based on CBC measurements (CBC model) in classifying cats into those with very low or increased risk of FP was assessed by computing the area under ROC curve (AUROC). AUROC was interpreted as follows: $\geq 90 \%$ - an excellent test, $80-89 \%$ - a good test, $70-79 \%$ - a fair test, $<70 \%$ - a poor test [24] and compared using the nonparametric method [45]. For the optimal cut-off value diagnostic sensitivity (Se), diagnostic specificity (Sp) as well as positive and negative likelihood ratios (LR+ and LR-) were calculated to evaluate diagnostic usefulness of CBC model. The 95\% confidence intervals (CI 95\%) for proportions and LRs were calculated using the Wilson score method and log method, respectively [46]. A significance level $(\alpha)$ was set at 0.05 . All statistical tests were two-sided. The statistical analysis was performed in TIBCO Statistica 13.3 (TIBCO Software Inc., Palo Alto, CA, USA) and IBM SPSS Statistics 26 (IBM Corporation, Armonk, NY, USA).

All methods were carried out in accordance with relevant guidelines and regulations.

\section{Abbreviations}

a: Significance level; AUROC: Area under ROC curve; AUS: Abdominal ultrasonography; BNC: Band neutrophil count; CBC: Complete blood count; Cl 95\%: 95\% confidence interval; DGGR: 1,2-o-Dilauryl-Rac-Glycero-3-Glutaric Acid-(6'-Methylresorufin) Ester; FP: Feline pancreatitis; fPLI: Feline pancreatic lipase immunoreactivity; H\&L $X^{2}$ : Hosmer \& Lemeshow chi-square test; IQR: Interquartile range; $L R+$ : Positive likelihood ratio (likelihood ratio of a positive result); LR-: Negative likelihood ratio (likelihood ratio of a negative result); NLR: Neutrophil-to-lymphocyte ratio; NPV: Negative predictive value; PPV: Positive predictive value; ROC: Receiver operating characteristic; Se: Diagnostic sensitivity; Sp: Diagnostic specificity; WBC: Total leukocyte count (white blood cell count).

\section{Supplementary Information}

The online version contains supplementary material available at https://doi. org/10.1186/s12917-021-03098-z.

Additional file 1. Numerical values of 7 complete blood count (CBC) measurements in 73 lethargic and anorectic and the cut-off values used for their categorization based on the highest Youden's index (J).

Acknowledgments

We are indebted to the owners of veterinary clinics and the owners of cats for their assistance and cooperation.

\section{Authors' contributions}

MMK, MC, and JK designed research; MMK, OS-J, AM, MM, and MC performed research; MMK and MC performed statistical and epidemiological analysis. MMK and MC wrote the draft manuscript. JK and MC edited and reviewed the manuscript. All authors read and approved the final manuscript.

Funding

Not applicable.

Availability of data and materials

The database is available from authors on request.

\section{Declarations}

\section{Ethics approval and consent to participate}

No ethics commission approval for this study was required according to Polish legal regulations (the Act on the Protection of Animals Used for Scientific or Educational Purposes of 15 January 2015) as only routine diagnostic procedures essential given the clinical status of the cat were performed and the study was purely analytical. An informed consent of each cat's owner for participation in the study was obtained. 


\section{Consent for publication \\ Not applicable.}

\section{Competing interests}

The authors declare that they have no competing interests.

\section{Author details}

${ }^{1}$ Division of Veterinary Epidemiology and Economics, Institute of Veterinary Medicine, Warsaw University of Life Sciences-SGGW, Nowoursynowska 159c 02-776 Warsaw, Poland. ${ }^{2}$ Department of Small Animal Diseases with Clinic, Institute of Veterinary Medicine, Warsaw University of Life Sciences-SGGW, Nowoursynowska 159c, 02-776 Warsaw, Poland.

\section{Received: 26 April 2021 Accepted: 29 November 2021} Published online: 11 December 2021

\section{References}

1. Simpson KW. The emergence of feline pancreatitis. J Vet Intern Med. 2001;15:327-8.

2. Xenoulis PG. Diagnosis of pancreatitis in dogs and cats. J Small Anim Pract. 2015;56:13-26.

3. Xenoulis PG, Steiner JM. Current concepts in feline pancreatitis. Top Companion Anim Med. 2008;23:185-92.

4. Bazelle J, Watson P. Is it being overdiagnosed? Feline pancreatitis. Vet Clin North Am Small Anim Pract. 2020;50:1107-21.

5. Forman MA, Steiner JM, Armstrong PJ, Camus MS, Gaschen L, Hill SL, et al. ACVIM consensus statement on pancreatitis in cats. J Vet Intern Med. 2021;35:703-23

6. Hill RC, Van Winkle TJ. Acute necrotizing and acute suppurative pancreatitis in the cat: a retrospective study of 40 cases (1976-1989). J Vet Intern Med. 1993;7:25-33.

7. De Cock HE, Forman MA, Farver TB, Marks SL. Prevalence and histopathologic characteristics of pancreatitis in cats. Vet Pathol. 2007:44:39-49.

8. Ferreri JA, Hardam E, Kimmel SE, et al. Clinical differentiation of acute necrotizing from chronic nonsuppurative pancreatitis in cats: 63 cases (1996-2001). J Am Vet Med Assoc. 2003;223:469-74.

9. Xenoulis PG, Steiner JM. Canine and feline pancreatic lipase immunoreactivity. Vet Clin Pathol. 2012;41:312-24.

10. Forman MA, Marks SL, De Cock HE, Hergesell EJ, Wisner ER, Baker TW, et al. Evaluation of serum feline pancreatic lipase immunoreactivity and helical computed tomography versus conventional testing for the diagnosis of feline pancreatitis. J Vet Intern Med. 2004;18:807-15.

11. Forman MA, Shiroma J, Armstrong PJ, et al. Evaluation of feline pancreasspecific lipase (Spec $\mathrm{PPL}^{\mathrm{TM}}$ ) for the diagnosis of feline pancreatitis [abstract]. J Vet Intern Med. 2009;23:733-4.

12. Oppliger S, Hartnack S, Riond B, Reusch CE, Kook PH. Agreement of the serum Spec $\mathrm{fPL}^{\mathrm{TM}}$ and 1,2-o-dilauryl-rac-glycero-3-glutaric acid-(6'methylresorufin) ester lipase assay for the determination of serum lipase in cats with suspicion of pancreatitis. J Vet Intern Med. 2013:27:1077-82.

13. Oppliger S, Hilbe M, Hartnack S, Zini E, Reusch CE, Kook PH. Comparison of serum Spec $\mathrm{fPL}\left({ }^{\mathrm{TM}}\right)$ and 1,2-o-dilauryl-rac-glycero-3-glutaric acid-(6'methylresorufin) ester assay in 60 cats using standardized assessment of pancreatic histology. J Vet Intern Med. 2016;30:764-70.

14. Törner K, Staudacher M, Tress U, Weber CN, Stadler C, Grassinger JM, et al. Histopathology and feline pancreatic lipase immunoreactivity in inflammatory, hyperplastic and neoplastic pancreatic diseases in cats. J Comp Pathol. 2020;174:63-72.

15. Gerhardt A, Steiner JM, Williams DA, Kramer S, Fuchs C, Janthur M, et al. Comparison of the sensitivity of different diagnostic tests for pancreatitis in cats. J Vet Intern Med. 2001:15:329-33.

16. Williams JM, Panciera DL, Larson MM, Werre SR. Ultrasonographic findings of the pancreas in cats with elevated serum pancreatic lipase immunoreactivity. J Vet Intern Med. 2013;27:913-8.

17. Lidbury JA, Suchodolski JS. New advances in the diagnosis of canine and feline liver and pancreatic disease. Vet J. 2016;215:87-95.

18. Conceição MEBAMD, Uscategui RAR, Bertolo PHL, de Souza DC, Rolemberg DDS, de Moraes PC, et al. Assessment of postoperative inflammatory markers and pain in cats after laparoscopy and miniceliotomy ovariectomy. Vet Rec. 2018;183:656.
19. Hodgson N, Llewellyn EA, Schaeffer DJ. Utility and prognostic significance of neutrophil-to-lymphocyte ratio in dogs with septic peritonitis. J Am Anim Hosp Assoc. 2018:54:351-9.

20. Pierini A, Gori E, Lippi I, Ceccherini G, Lubas G, Marchetti V. Neutrophilto-lymphocyte ratio, nucleated red blood cells and erythrocyte abnormalities in canine systemic inflammatory response syndrome. Res Vet Sci. 2019:126:150-4.

21. Chiti LE, Martano M, Ferrari R, Boracchi P, Giordano A, Grieco V, et al. Evaluation of leukocyte counts and neutrophil-to-lymphocyte ratio as predictors of local recurrence of feline injection site sarcoma after curative intent surgery. Vet Comp Oncol. 2020;18:105-16.

22. Neumann S. Neutrophil-to-lymphocyte and platelet-to-lymphocyte ratios in dogs and cats with acute pancreatitis. Vet Clin Pathol. 2021:50:45-51.

23. Petrucci GN, Lobo L, Queiroga F, Martins J, Prada J, Pires I, et al. Neutrophil-to-lymphocyte ratio is an independent prognostic marker for feline mammary carcinomas. Vet Comp Oncol. 2021;19:482-91.

24. Carter JV, Pan J, Rai SN, Galandiuk S. ROC-ing along: evaluation and interpretation of receiver operating characteristic curves. Surgery. 2016:159:1638-45.

25. Thrusfield M. Veterinary epidemiology. 4th ed. Chichester: Wiley; 2018. p. 429-56.

26. Knottenbelt CM, Blackwood L. The blood. In: Chandler EA, Gaskell CJ, Gaskel RM, editors. Feline medicine and therapeutics. 3rd ed. Ames: Blackwell Publishing; 2007. p. 235-56.

27. Paltrinieri S. The feline acute phase reaction. Vet J. 2008;177:26-35.

28. Declue AE, Delgado C, Chang CH, Sharp CR. Clinical and immunologic assessment of sepsis and the systemic inflammatory response syndrome in cats. J Am Vet Med Assoc. 2011:238:890-7.

29. Thrall MA, Weiser G, Allison RW, Campbell TW. Veterinary hematology and clinical chemistry. 2nd ed. Baltimore: Lippincott Williams \& Wilkins; 2004. p. 120-45.

30. Hoshiya T, Watanabe D, Matsuoka T, Horiguchi K, Miki Y, Mizuguchi H, et al. Acute phase response in toxicity studies. II. Findings in beagle dogs injected with endotoxin or subjected to surgical operation. J Toxicol Sci. 2001:26:103-9.

31. Rosenfeld AJ, Dial SM. Clinical pathology for the veterinary team. Ames: Wiley-Blackwell; 2010. p. 61-71.

32. Huang Z, Fu Z, Huang W, Huang K. Prognostic value of neutrophilto-lymphocyte ratio in sepsis: a meta-analysis. Am J Emerg Med. 2020;38:641-7

33. McGee S. Simplifying likelihood ratios. J Gen Intern Med. 2002:17:646-9.

34. Deeks JJ, Altman DG. Diagnostic tests 4: likelihood ratios. BMJ. 2004;329:168-9.

35. Fagan TJ. Letter: Nomogram for Bayes theorem. N Engl J Med. 1975:293:257.

36. Caraguel CG, Vanderstichel R. The two-step Fagan's nomogram: ad hoc interpretation of a diagnostic test result without calculation. Evid Based Med. 2013;18:125-8.

37. Begg CB. Biases in the assessment of diagnostic tests. Stat Med. 1987:6:411-23

38. Oppliger S, Hartnack S, Reusch CE, Kook PH. Agreement of serum feline pancreas-specific lipase and colorimetric lipase assays with pancreatic ultrasonographic findings in cats with suspicion of pancreatitis: 161 cases (2008-2012). J Am Vet Med Assoc. 2014;244:1060-5.

39. Paran E, Hugonnard M. Agreement of feline and canine pancreas-specific lipase with pancreatic ultrasonographic findings in 62 cats and 54 dogs with suspicion of pancreatitis: a retrospective study (2007-2013) [abstract]. J Vet Intern Med. 2017;31:261-2.

40. Rutjes AW, Reitsma JB, Coomarasamy A, Khan KS, Bossuyt PM. Evaluation of diagnostic tests when there is no gold standard. A review of methods. Health Technol Assess. 2007:11:iii ix-51.

41. Staquet M, Rozencweig M, Lee YJ, Muggia FM. Methodology for the assessment of new dichotomous diagnostic tests. J Chronic Dis. 1981;34:599-610

42. Stockham SL, Scott MA. Fundamentals of veterinary clinical pathology. 2nd ed. Oxford: Blackwell Publishing; 2008. p. 68.

43. Brady CA, Otto CM, Van Winkle TJ, King LG. Severe sepsis in cats: 29 cases (1986-1998). J Am Vet Med Assoc. 2000;217:531-5.

44. Hosmer DW, Lemeshow S. Applied logistic regression. 2nd ed. New Jersey: Wiley; 2000. p. 31-46. 
45. DeLong ER, DeLong DM, Clarke-Pearson DL. Comparing the areas under two or more correlated receiver operating characteristic curves: a nonparametric approach. Biometrics. 1988;44:837-45.

46. Altman D, Machin D, Bryant T, Gardner M. Statistics with confidence:

confidence intervals and statistical guidelines. 2nd ed. Bristol: BMJ Books; 2000. p. 46-7. 108-10

\section{Publisher's Note}

Springer Nature remains neutral with regard to jurisdictional claims in pub-

lished maps and institutional affiliations.

- fast, convenient online submission

- thorough peer review by experienced researchers in your field

- rapid publication on acceptance

- support for research data, including large and complex data types

- gold Open Access which fosters wider collaboration and increased citations

- maximum visibility for your research: over 100M website views per year

At $\mathrm{BMC}$, research is always in progress.

Learn more biomedcentral.com/submissions 